\title{
Factors associated with depressive symptoms among the elderly in China: structural equation model
}

\author{
Yaofei Xie, ${ }^{1, *}$ Mengdi Ma, ${ }^{2, *}$ Wenwen Wu, ${ }^{1}$ Yupeng Zhang, ${ }^{1}$ Yuting Zhang, ${ }^{1}$ and \\ Xiaodong $\operatorname{Tan}^{1}$ \\ ${ }^{1}$ School of Health Sciences, Wuhan University, Wuhan, Hubei Province, China \\ ${ }^{2}$ Wuhan Blood Center, Wuhan, Hubei Province, China
}

Objectives: To establish a structural equation model for exploring the direct and indirect relationships of depressive symptoms and their associated factors among the Chinese elderly population.

Design: A cross-sectional research. The 2015 data from the China Health and Retirement Longitudinal Study (CHARLS) were adopted.

Setting: CHARLS is an ongoing longitudinal study assessing the social, economic, and health status of nationally representative samples of middle-aged and elderly Chinese residents.

Participants: A total of 5791 participants aged 60 years and above were included.

Measurements: Depressive symptoms were used as the study outcome. Sociodemographic characteristics, poor health status, unhealthy habits, and sleep duration were used as predictors. Confirmatory factor analysis was first conducted to test the latent variables. Structural equation model was then utilized to examine the associations among latent variables and depressive symptoms.

Results: The mean age of the participants was $68.82 \pm 6.86$ years, with $55.53 \%$ being males. The total prevalence of depressive symptoms was $37.52 \%$. The model paths indicated that sociodemographic characteristics, poor health status, unhealthy habits, and sleep duration were directly associated with depressive symptoms, and the effects were $0.281,0.509,-0.067$, and -0.162 , respectively. Sociodemographic characteristics, unhealthy habits, and sleep duration were indirectly associated with depressive symptoms, mediating by poor health status. Their effects on poor health status were $-0.093,0.180$, and -0.279 , respectively. All paths of the model were significant $(P<0.001)$. The model could explain $40.9 \%$ of the variance in the depressive symptoms of the Chinese elderly population.

Conclusions: Depressive symptoms were significantly associated with sociodemographic characteristics, poor health status, unhealthy habits, and sleep duration among Chinese elderly population. The dominant predictor of depressive symptoms was poor health status. Targeting these results might be helpful in rationally allocating health resources during screening or other mental health promotion activities for the elderly.

Key words: aged, Chinese, depression, mental health

\section{Introduction}

China is experiencing a rapid and intense aging process and has the largest aging population in the world. By the end of 2018, its population with elderly aged 60 years or above (locally defined age cutoff for the elderly) reached 249.49 million, accounting for

Correspondence should be addressed to: Dr. Xiaodong Tan, Add.: No.115 of Donghu Road, Wuhan 430000, China. Phone: + 8613507135465. Email: 00300469@whu.edu.cn. Received 19 Mar 2020; revision requested 03 May 2020; revised version received 29 May 2020; accepted 20 Jun 2020. First published online 04 August 2020.

*These authors contributed equally to this work.
$17.9 \%$ of the total population (National Bureau of Statistics, 2019). The expansion of the elderly population, especially older seniors, likely causes a substantial increase in nonproductive consumption expenditures, such as medical treatment, nursing, and old age security. Therefore, healthy aging is crucial to sustainable development.

Depression (major depressive disorders) is a major public health issue affecting the health of the elderly population in China. According to a nationwide survey, China Health and Retirement Longitudinal Study (CHARLS), about $40 \%$ 
of older adults reported depressive symptoms (CHARLS Research Team, 2013). In addition, the proportion of "empty-nesters," i.e. those who were not living with their children, has increased remarkably, and the number is still rising (Gao et al., 2017). This group is more likely to suffer from mental health problems (Su et al., 2018). In contrast to depression in the younger population, depression in late life is associated with decreased physical, cognitive, and social functioning and increased self-neglect $(\mathrm{Ni}$ et al., 2017). Moreover, depression in late life can lead to chronic disease course, poor response to antidepressants, and high relapse rate, which are associated with increased mortality (Van Orden et al., 2015; Haigh et al., 2018). Consequently, the issue of depression among Chinese elderly population should be settled urgently.

Some sociodemographic factors, such as gender, age, marital status, place of residence, employment status, and educational level, have been reported to be associated with depression in the elderly population (Chin et al., 2016; Yang et al., 2017; Cao et al., 2016). Negative life events, multiple chronic health conditions, major physical diseases, and poor selfrated general health in older people can also increase the risk of depression (Alexandrino-Silva et al., 2011). However, studies have yet to adopt a structural equation model (SEM) for exploring the relationships between factors and depressive symptoms, which are the major manifestations of depressive disorders. SEM is a multivariate analysis technique used to ascertain relationships among variables and provide a comprehensive and flexible approach for data analysis (Crowley and Fan, 1997). It enables the simultaneous estimation of structural relationships among multiple independent and dependent variables while controlling measurement errors; it can also identify direct, indirect, and total effects among variables (Pentz and Chou, 1994). Given these advantages, this study aimed to establish an SEM to analyze the structural relationships of depressive symptoms and associated factors among the elderly population in China.

\section{Methods}

\section{Sample}

The publicly available data of CHARLS collected in 2015 were used. CHARLS is an ongoing longitudinal study assessing the social, economic, and health status of nationally representative samples of middle-aged and elderly Chinese residents. In CHARLS, a multi-stage stratified probabilityproportional-to-size (PPS) sampling method is adopted, and the software package CHARLS-GIS is created to make village sampling frames. Data were collected through face-to-face interviews by trained investigators. The response rate was over $85 \%$ (CHARLS, 2018). After participants younger than 60 years and participants with missing values on the main outcome variables were removed from the study, a total of 5791 samples were retained.

\section{Study variables}

Based on the content of "CHARLS Followup Questionnaire 2015" (CHARLS, 2018) and our literature review, 16 observational variables were included in this study, and we hypothesized that four latent variables could be extracted and used to collectively reflect the sociodemographic characteristics, poor health status, unhealthy habits, and sleep duration of the participants. The hypothetical latent variables and the corresponding observational variables are presented in Supplementary Table 1. For the sociodemographic characteristic factor, household consumption expenditure in the previous year rather than household income was used to reflect the economic status of the participants because it was a better representation of the permanent income of the retired and the elderly (Islam and Smyth, 2015). For the poor health status factor, the activity of daily living scale, which was recommended by the World Health Organization for assessing the health status of the elderly population, was used to assess the participants' ability to perform daily living activities (Katz et al., 1963).

\section{Assessment of depressive symptoms}

Depressive symptoms were quantified using the 10 -item version of the Center for Epidemiologic Studies Depression Scale (CES-D-10), which accurately predicts depressive symptoms (Andresen et al., 1994). The Chinese version of this scale has good reliability and validity among the elderly population in China, and Cronbach's alpha was 0.813 (Huang et al., 2015).

The 10 items were as follows: item 1, "I was bothered by things that do not usually bother me;" item 2, "I had trouble keeping my mind on what I was doing;" item 3, "I felt depressed;" item 4, "I felt everything I did was an effort;" item 5, "I felt hopeful about the future;" item 6, "I felt fearful;" item 7, "My sleep was restless;" item 8, "I was happy;" item 9, "I felt lonely;" and item 10, "I could not get going." Each item was rated on a 3-point Likert scale with answers varying from "rarely or none of the time ( $<1$ day)" to "most or all of the time (5-7 days)." Thus, the total score of the CES-D-10 scale ranged from 0 to 30 . A score of 10 or higher indicated the presence of significant depressive symptoms.

\section{Statistical analysis}

Stata/SE version 15.1 was used for preliminary data processing and descriptive statistics of the 
demographic variables and depressive symptoms. SEM was created by using Mplus version 7.4 to test the hypothetical associations among the variables. Confirmatory factor analysis (CFA) was first conducted to test whether the latent variables could be well reflected by the involved observational variables. Based on the measurement model, SEM was then utilized to examine the associations among latent variables and depressive symptoms.

All continuous variables were converted to categorical variables before they were analyzed. The correlation matrix of the study variables was examined using Pearson's correlation coefficients. Weighted least squares with the mean and variance (WLSMV)-adjusted method were used for the parameter estimation of CFA and SEM. All the effects were quantified with standardization, and two-sided $P<0.05$ was regarded as statistically significant. Four goodness-of-fit indices: maximum likelihood chi-square $\left(\chi^{2}\right)$ and degrees of freedom $(d f)$, comparative fit index (CFI), TuckerLewis index (TLI), and root mean square error of approximation (RMSEA) were adopted to check whether the hypothesized model was well fitted to the data. The value of $\chi^{2}$ is susceptible to sample size, and it is statistically significant for models with large sample size (Bollen and Long, 1993). The value of CFI or TLI for a good fitting model should be 0.90 or greater ( $\mathrm{Hu}$ and Bentler, 1999). And a model is considered excellent fit when RMSEA value is less than 0.05 (Bollen and Long, 1993).

\section{Results}

\section{Participants and depressive symptoms}

Of the 5791 participants, $55.53 \%$ were males $(n=3216)$, and their mean age was 68.82 years (standard deviation, 6.86; range, 60-102). Nearly two-thirds of the participants were living with their spouses, and the remaining participants were married but were temporarily not living with their spouses, separated, divorced, widowed, or never married. Most of the participants resided in a village. Nearly half of the participants' educational levels were elementary school or below, followed by illiterate. More than half of the participants reported that their household consumption expenditure per capita was within 2,000 RMB. The detailed sociodemographic characteristics and corresponding prevalence of the depressive symptoms of the participants are summarized in Table 1 . As shown in Table 1, the total prevalence of depressive symptoms was $37.52 \%$. The participants with different genders, marital status, residence, and educational levels showed a significant difference in the prevalence of depressive symptoms $(P<0.001)$.

\section{Measurement model construction}

All hypothetical latent variables, namely, sociodemographic characteristics, poor health status, unhealthy habits, and sleep duration, were specified in the measurement model. Observational variables with insignificant paths or low factor loadings were deleted from the initial measurement model to determine the most parsimonious model. After preliminary data analysis, 11 observational variables were retained, and their assignment is illustrated in Supplementary Table 2. The correlation matrix of the study variables is presented in Table 2. Many of the variables showed significant correlations.

Figure 1 shows the final measurement model. Sociodemographic characteristics, poor health status, unhealthy habits, and sleep duration were assigned three, four, two, and two observational variables, and the factor loadings ranged $0.457-0.687,0.491-$ $0.863,0.575-0.747$, and $0.224-0.695$, respectively. All these factor loadings were significant $(P<0.001)$. The four latent variables were significantly intercorrelated. Among them, sociodemographic characteristics displayed the strongest association with unhealthy habits $(r=0.516)$; however, it was correlated with poor health status with a minimum correlation coefficient $(r=0.046)$. All the correlation paths were significant $(P<0.01)$.

The measurement model was well fitted to the data. The goodness-of-fit indices were as follows: $\chi^{2}=374.765, \quad d f=38, \quad P<0.001, \quad$ CFI $=0.960$, $\mathrm{TLI}=0.942$, and RMSEA $=0.039(95 \%$ confidence intervals $[\mathrm{CI}]=0.036-0.043)$.

\section{SEM}

Figure 2 displays the SEM designed on the basis of the measurement model. Sociodemographic characteristics, poor health status, unhealthy habits, and sleep duration had direct paths to depressive symptoms, and their regression coefficients were 0.281 , $0.509,-0.067$, and -0.162 , respectively. Sociodemographic characteristics, unhealthy habits, and sleep duration also had direct paths to poor health status, and these variables were correlated with one another. All the parameters were significant $(P<0.01)$. The model was also well fitted to the data: $\chi^{2}=513.471, d f=45, P<0.001, \mathrm{CFI}=0.957, \mathrm{TLI}=$ 0.937 , and $\mathrm{RMSEA}=0.042 \quad(95 \% \quad \mathrm{CI}=0.039$ 0.046).

Table 3 illustrates the direct and indirect associations of all latent variables with depressive symptoms. Among the four latent variables, poor health status had the strongest direct and total associations with depressive symptoms. According to the SEM, the identified variables explained $40.9 \%$ of the variance in depressive symptoms. 
Table 1. Sociodemographic characteristics of participants and depressive symptoms

\begin{tabular}{|c|c|c|c|}
\hline VARIABLES & $\mathrm{N}(\%)$ & $\begin{array}{l}\text { DEPRESSIVE SYMPTOMS } \\
\text { (N/PREVALENCE \%) }\end{array}$ & $\chi^{2}$ \\
\hline Gender & & & $157.471^{* * *}$ \\
\hline Male & $3216(55.53)$ & $977(30.38)$ & \\
\hline Female & $2575(44.47)$ & $1196(46.45)$ & \\
\hline Age (years old) & & & 2.646 \\
\hline $60-69$ & $3504(60.51)$ & $1318(37.61)$ & \\
\hline $70-79$ & $1791(30.93)$ & $685(38.25)$ & \\
\hline$\geq 80$ & $496(8.57)$ & $170(34.27)$ & \\
\hline Marital status & & & $83.448^{* * *}$ \\
\hline Living with spouse present & $3788(65.41)$ & $1262(33.32)$ & \\
\hline Married but not living with spouse temporarily & $190(3.28)$ & $81(42.63)$ & \\
\hline Separated/Divorced/Widowed/Never married & $1813(31.31)$ & $830(48.78)$ & \\
\hline Residence & & & $102.656^{* * *}$ \\
\hline Main city zone & $916(15.82)$ & $226(24.67)$ & \\
\hline Town area & $540(9.32)$ & $167(30.93)$ & \\
\hline Township & $255(4.40)$ & $88(34.51)$ & \\
\hline Village & $4080(70.45)$ & $1692(41.47)$ & \\
\hline Educational level & & & $190.701^{* * *}$ \\
\hline Illiterate & $1705(29.44)$ & $812(47.62)$ & \\
\hline Elementary school or below & $2706(46.73)$ & $1030(38.06)$ & \\
\hline Middle school & $851(14.70)$ & $229(26.91)$ & \\
\hline High school or Vocational school & $410(7.08)$ & $81(19.76)$ & \\
\hline Bachelor's degree/Associate degree or greater & $119(2.05)$ & $21(17.65)$ & \\
\hline Household per capita consumption expenditure (RMB) & & & 3.474 \\
\hline$\leq 2,000$ & $3302(57.02)$ & $1233(37.34)$ & \\
\hline$\sim 5,000$ & $1233(21.29)$ & $487(39.50)$ & \\
\hline$\sim 10,000$ & $630(10.88)$ & $223(35.40)$ & \\
\hline$>10,000$ & $626(10.81)$ & $230(36.74)$ & \\
\hline Total & $5791(100.00)$ & $2173(37.52)$ & \\
\hline
\end{tabular}

${ }^{*} P<0.05 ;{ }^{* *} P<0.01 ;{ }^{* * *} P<0.001$

\section{Discussion}

Depression is one of the most common mental disorders in the elderly, and this condition can affect their physical and mental health, leading to the decline in the quality of life and even death (Doraiswamy et al., 2002). This study is the first to explore the structural relationships of depressive symptoms with the possible associated factors among the elderly population in China. The model indicated that sociodemographic characteristics, poor health status, unhealthy habits, and sleep duration had significant direct relationships with depressive symptoms. Sociodemographic characteristics, unhealthy habits, sleep duration, and depressive symptoms were also indirectly related. The direct and indirect paths suggested that poor health status was a dominant predictor of depressive symptoms.

\section{Sociodemographic characteristics}

The sociodemographic characteristics in the present study included three observed variables: age, marital status, and educational level. In terms of age, the paths indicated that older participants were prone to depressive symptoms. This result was consistent with a few studies on elderly population (Glaesmer et al., 2011; Urbina Torija et al., 2007; Kaneko et al., 2007). Unlike the young, the prevalence of depressive symptoms generally increases with age among the elderly population. This difference may be mainly attributed to the lower physical and cognitive functions, which are closely related to depressive symptoms among older people (Al-Otaibi et al., 2007). In terms of marital status, our results suggested that older people who were not living with their spouses (separated/divorced/widowed/single) had relatively high odds of depressive symptoms. Similarly, many studies have reported that marital status is a key variable that affects depressive symptoms among the elderly population (Javed, 2014; Xie et al., 2010; Fatima et al., 2019). Kim and McKenry (2002) found that separation, divorce, and death of a spouse have significant effects on exacerbating depressive symptoms. Elderly people have relatively small social support networks, and their spouses are an important channel for them to 


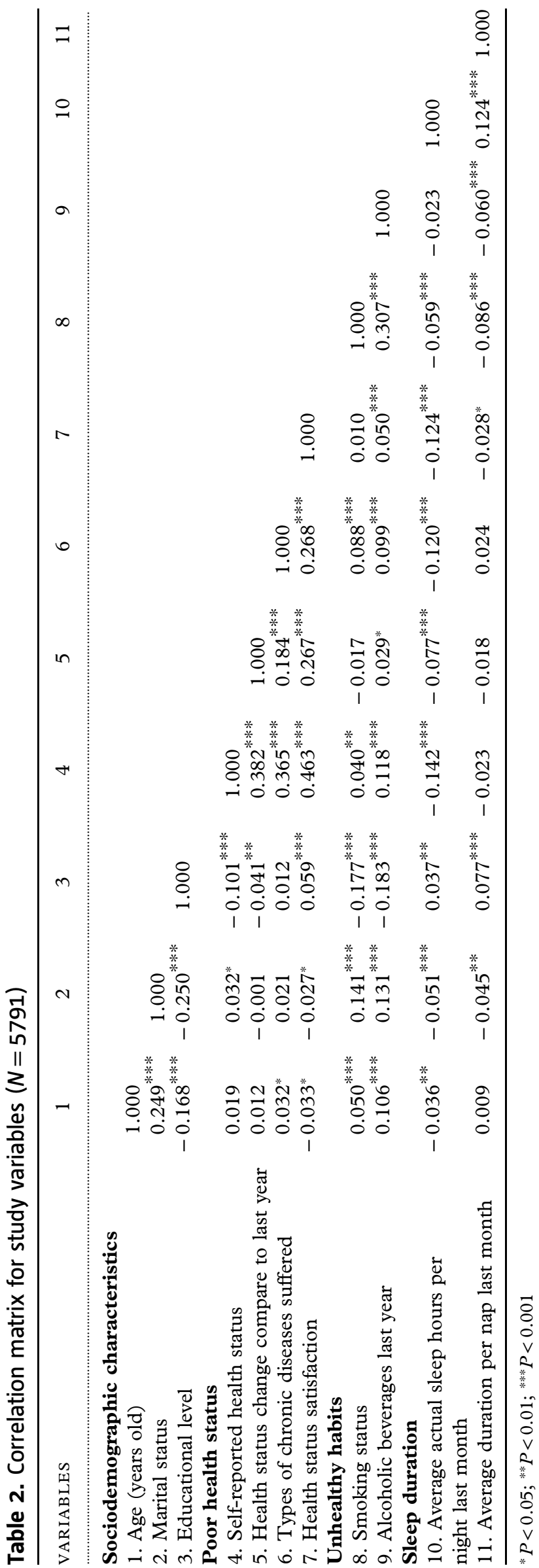

gain support and eliminate negative emotions. Therefore, elderly people not living with their spouses are more likely to feel lonely and be affected by adverse events. In terms of educational level, the results indicated that educational level could clearly predict depressive symptoms among the elderly population, and this observation was consistent with previous findings (Gibson et al., 2013; Zhang et al., 2019; de la Torre-Luque et al., 2019). This may be due to the relatively low social and economic status of poorly educated elderly; therefore, they were more likely to have low self-evaluation and negative emotions. Moreover, highly educated elderly people tended to have more knowledge and an active mind, which enabled them to better channel negative emotions and pressure.

\section{Poor health status}

Our results suggested that poor health status was a strong predictor of depressive symptoms among the elderly people in China. A prior study in Taiwan (Hsiao and Chen, 2018) indicated that the number of chronic diseases and the self-assessed health status are the factors significantly associated with depressive symptoms among the elderly residents of care settings Tanaka et al. (2011) also reported that having a chronic disease increases the risk of depressive symptoms in the elderly. In fact, some common physical disorders are risk factors of depressive symptoms in the elderly population (Weinger and Lee, 2006; Hackett et al., 2005). These disorders could cause indisposition and decline of physical function, which could seriously affect the quality of life of the elderly and cause or exacerbate depressive symptoms to some extent. The economic and psychological burden brought by this disease could simultaneously cause depressive symptoms. Consequently, the elderly who had a poor physical health status needed more attention.

\section{Unhealthy habits}

For unhealthy habits, the direct association between smoking and depressive symptoms should be interpreted with caution. Previous population-based studies reported inconsistent findings: two crosssectional studies on the depressive symptoms of the elderly population in Brazil (Gazalle et al., 2004) and Iran. Taheri Tanjanai et al. (2017) found that the odds of having depressive symptoms in smokers are statistically higher than those in nonsmokers. A large population-based longitudinal study (Luijendijk et al., 2008) also found that current smoking status is independently associated with depressive symptoms. However, a cohort study on the associations of lifestyle factors with depressive symptoms in old Taiwanese people (Tsai et al., 2013) indicated that smoking 


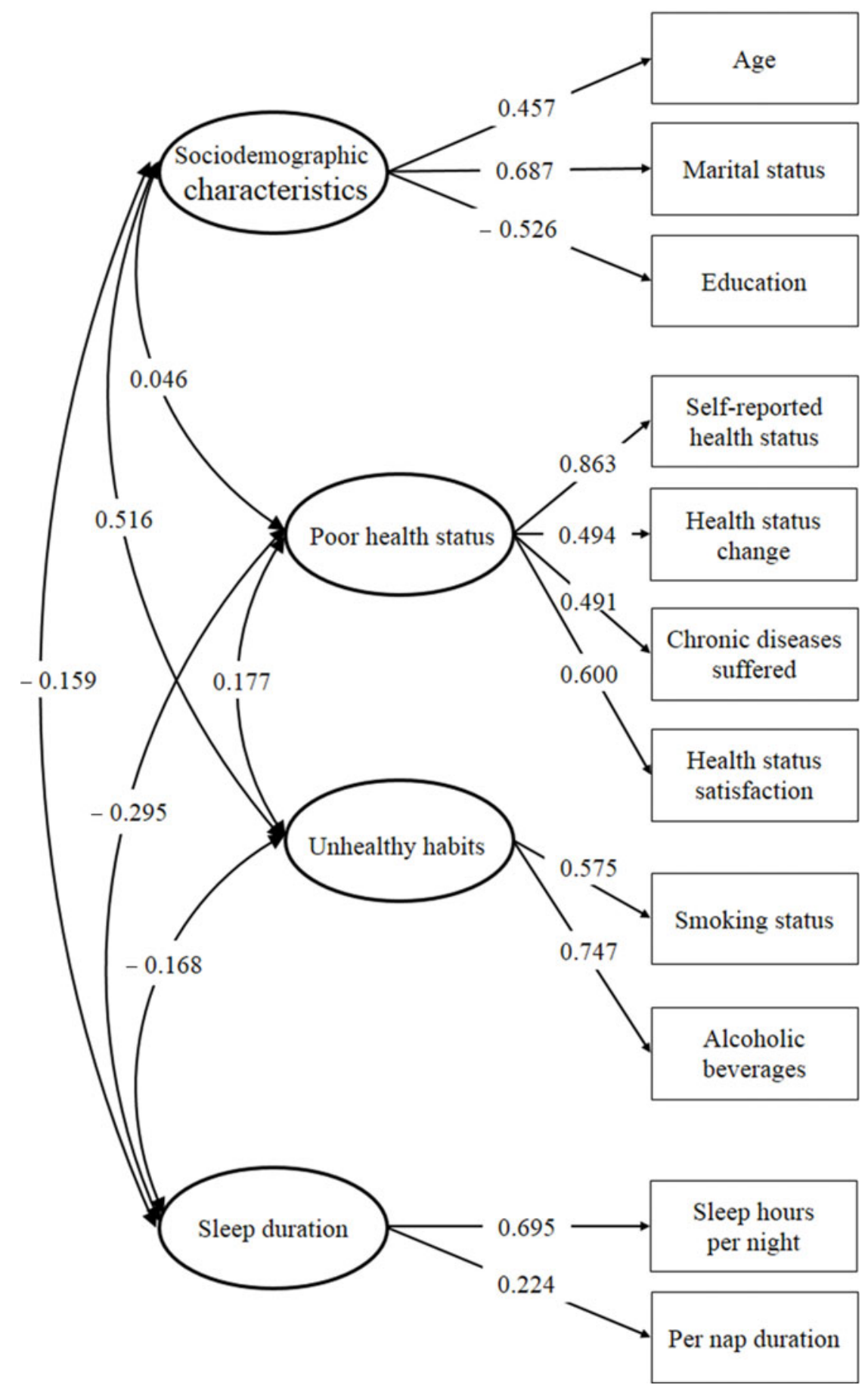

Figure 1. The measurement model of latent variables. Four latent variables and 11 manifest variables are connected by significant paths. The numbers on the straight arrows indicate the path coefficients. Every pair of latent variables is connected by bidirectional arrow curves, and the numbers on the lines indicate the correlation coefficients. The variances are set to 1.000 during the model estimation.

showed no significant associations with concurrent depressive symptoms, but it could increase the development of depressive symptoms. In the present study, smoking was negatively correlated with depressive symptoms, but their direct association was not particularly strong. This result might be due to that the elderly might use smoking to relax, as a form of self-medication or a way of coping with stress and anxiety (Cassidy et al., 2004). And neuropharmalogical effects of nicotine on neurotransmitters could also be associated with improved mood (Kendler et al., 1993). Another possible reason might be the increased pressure experienced by the elderly to stop smoking, especially when they were forced to quit smoking because of chronic disease or other diseases (Hyland et al., 2004). Overall, smoking 


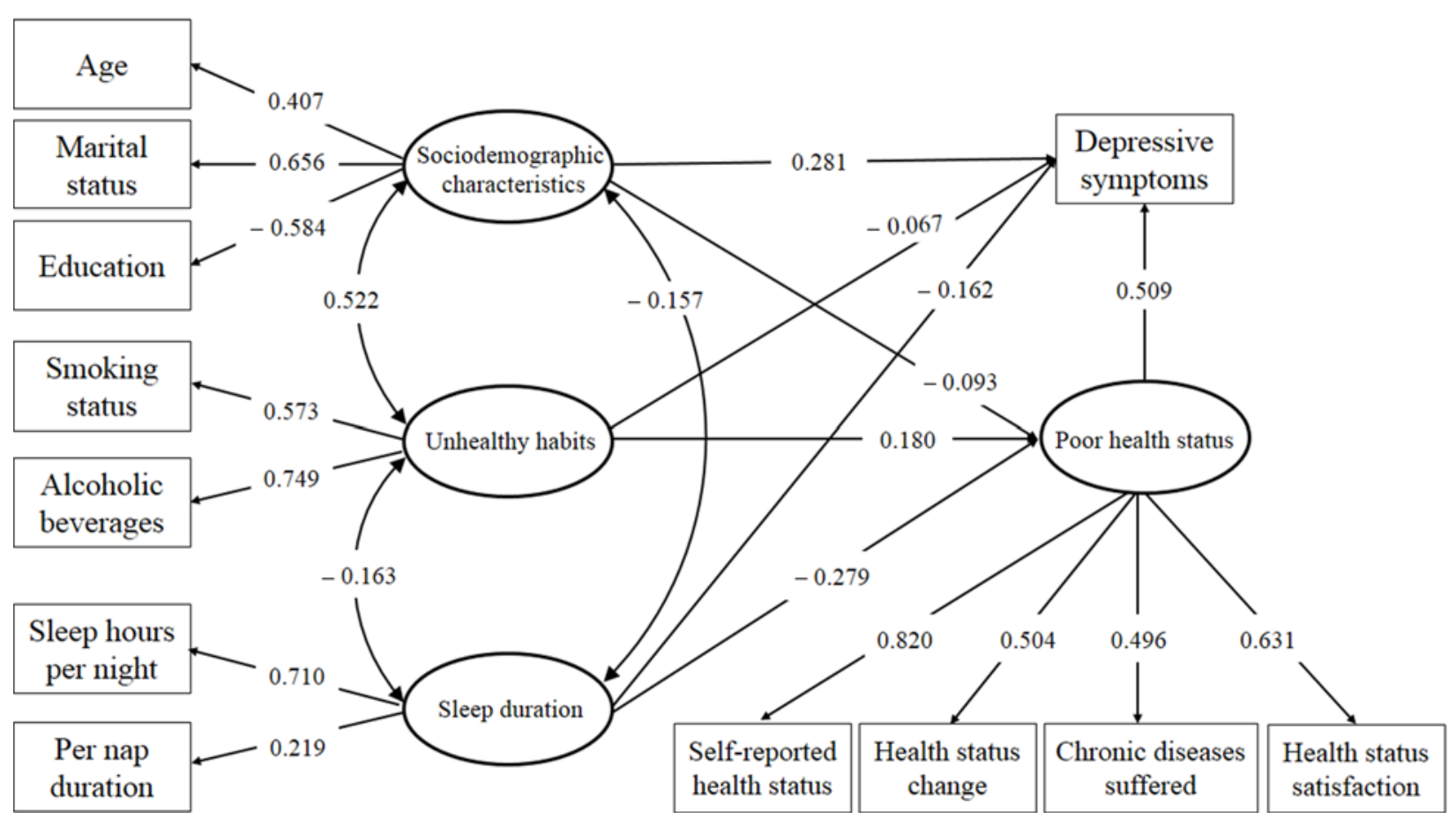

Figure 2. The structural equation model of sociodemographic characteristics, poor health status, unhealthy habits, sleep duration, and depressive symptoms. The relationships of four latent variables and their corresponding manifest variables and the associations of four latent variables and depressive symptoms are presented. The standardized coefficients are shown on the paths.

was still positively correlated with depressive symptoms because of its positive correlation with the poor health status variable. Thus, elderly population with depressive symptoms was more likely to report smoking.

Frequency of drinking also had a direct negative correlation with depressive symptoms in the present study. This result was similar to a prior study of rural elderly in China that reported alcohol consumption was associated with lower depressive symptoms scores (Gao et al., 2009). It might be explained by the link between moderate alcohol consumption and a more active and sociable lifestyle of the elderly (Hajat et al., 2004). And more social activity participation was considered a protective factor of depressive symptoms among the elderly (Borges et al., 2013; Dai et al., 2019; Yang et al., 2018). However, the direct negative correlation in this study needs to be read cautiously, because it was possible that the characteristics of heavy drinkers would be different. In fact, a few studies have reported that there was a J-shaped or U-shaped association between alcohol consumption and depressive symptoms among the elderly (Bots et al., 2008; Graham et al., 2007; Yun and Kim, 2020), namely, moderate alcohol consumption could lead to decreased levels of depressive symptoms, while heavy alcohol consumption was associated with an elevated risk of depressive symptoms. Thus, detailed quantifying alcohol consumption might be required to fully interpret the direct association between drinking and depressive symptoms among Chinese elderly population. Similar to smoking in present study, our results indicated that poor health status could act as a mediating effect between frequency of drinking and depressive symptoms among the elderly. This might be due to that alcohol problems could contribute to individual biological changes (Boden and Fergusson, 2011), and age-related physiological changes of the elderly could significantly increase the sensitivity and decrease tolerance to alcohol (Rodriguez et al., 2010), resulting in an impact on poor health status. This effect was consistent with the study of Solmi et al. that reported physical healthrelated quality of life could be a bridge node connecting depressive symptoms with alcohol consumption (Solmi et al., 2019). Overall, the frequency of drinking was positively correlated with depressive symptoms because of its harmfulness to health. This result was consistent with the results of studies carried out in Taiwan (Tsai et al., 2013) and Australia (Anstey et al., 2009).

\section{Sleep duration}

The association between sleep and depressive symptoms has been highlighted in other studies. Guimaraes et al. (2019) and Zalavadiya et al. (2017) reported that depressive symptoms in the elderly are associated with poor sleep quality. Our findings suggested 
Table 3. Standardized associations of latent variables on depressive symptoms

\begin{tabular}{|c|c|c|c|}
\hline \multirow[b]{2}{*}{ VARIABLES } & \multicolumn{3}{|c|}{ ASSOCIATION } \\
\hline & DIRECT & INDIRECT & TOTAL \\
\hline $\begin{array}{l}\text { Sociodemographic } \\
\text { characteristics }\end{array}$ & 0.281 & 0.134 & 0.415 \\
\hline Poor health status & 0.509 & 0 & 0.509 \\
\hline Unhealthy habits & -0.067 & 0.294 & 0.227 \\
\hline Sleep duration & -0.162 & -0.205 & -0.367 \\
\hline
\end{tabular}

that short sleep duration was associated with high rates of depressive symptoms. This result was consistent with the results of Wen et al. (2019), who found that elderly people with long sleep duration at night have a lower risk of developing depressive symptoms than those with short sleep duration. Sleep and depressive symptoms have a multifaceted interplay between them. Manber and Chambers (2009) reported that the risk of depressive symptoms in the group of the elderly with sleep disorders is statistically significant higher than that in the group without sleep disorders. In addition, depressive symptoms can cause and exacerbate sleep disorders in the elderly, and it is even the largest risk factor of sleep disorders (Staner, 2010; Ichimori et al., 2015).

\section{Strengths and limitations}

The strength of this study was the use of large-sample data, which were collected through face-to-face interviews. The influencing factors of depressive symptoms were mostly comprehensive, and the corresponding explanation might be incomplete with individual indicators. Therefore, our findings filled in the gap in research on the structural relationships of depressive symptoms and potential latent variables. Our results also simultaneously determined the direct and indirect connections between them.

However, some limitations should be considered in understanding the present results. First, the crosssectional sample of this study only allowed us to estimate associations between depressive symptoms and observed variables, so it could not establish causal relationships. Second, the result was based on the whole population. Thus, the differences between males and females and between urban and rural areas were not considered in our data analysis. Third, although the final SEM was adequately fitted to the data, more information and variables could be incorporated into the model for an enhanced data fitting and explanation of depressive symptoms. These issues could be addressed by conducting future longitudinal analysis to further understand the relationships of present observations and depressive symptoms. The inclusion of additional sample information and a more specific analysis should also be considered.

\section{Conclusion}

This study first established an SEM to explore the direct and indirect relationships of depressive symptoms and their associated factors in the Chinese elderly population. The results suggested that sociodemographic characteristics, poor health status, unhealthy habits, and sleep duration could appropriately predict depressive symptoms. Among these variables, poor health status was a major predictor. Sociodemographic characteristics, unhealthy habits, and sleep duration were also indirectly associated with depressive symptoms mediating by poor health status. These results indicated that health resources could be allocated rationally to enhance their utilization efficiency during depression screening and other public health actions.

\section{Conflict of interest}

None.

\section{Description of authors' roles}

Yaofei Xie conducted Mplus analysis of CFA and SEM and was a major contributor in writing the manuscript. Mengdi Ma interpreted the models, completed model fitting, and was a contributor in writing the manuscript. Wenwen Wu performed raw data processing. Yupeng Zhang and Yuting Zhang revised the manuscript. Xiaodong Tan designed and was responsible for the study. All authors read and approved the final manuscript.

\section{Acknowledgments}

The research team of CHARLS is acknowledged for their work.

\section{Supplementary material}

To view supplementary material for this article, please visit https://doi.org/10.1017/S1041610220001398

\section{References}

Al-Otaibi, B. et al. (2007). Depressive symptoms among Kuwaiti population attending primary healthcare setting: prevalence and influence of sociodemographic factors. Medical Principles and Practice, 16, 384-388. doi: 10.1159/ 000104813 
Alexandrino-Silva, C., Alves, T. F., Tofoli, L. F., Wang, Y. P. and Andrade, L. H. (2011). Psychiatry: life events and social support in late life depression. Clinics (Sao Paulo), 66, 233-238. doi: 10.1590/s180759322011000200009

Andresen, E. M., Malmgren, J. A., Carter, W. B. and Patrick, D. L. (1994). Screening for depression in well older adults: evaluation of a short form of the CES-D (Center for Epidemiologic Studies Depression Scale). American fournal of Preventive Medicine, 10, $77-84$.

Anstey, K. J. et al. (2009). Cardiovascular risk factors and life events as antecedents of depressive symptoms in middle and early-old age: PATH Through Life Study. Psychosomatic Medicine, 71, 937-943. doi: 10.1097/PSY $.0 \mathrm{~b} 013 \mathrm{e} 3181$ beab60

Boden, J. M. and Fergusson, D. M. (2011). Alcohol and depression. Addiction, 106, 906-914. doi: 10.1111/j.13600443.2010.03351.x

Bollen, K. A. and Long, J. S. (1993). Testing structural equation models. Newbury Park, CA: Sage Publications.

Borges, L. J., Benedetti, T. R., Xavier, A. J. and D'orsi, E. (2013). Associated factors of depressive symptoms in the elderly: EpiFloripa study. Revista Saude Publica, 47, 701-710. doi: 10.1590/S0034-8910.2013047003844

Bots, S., Tijhuis, M., Giampaoli, S., Kromhout, D. and Nissinen, A. (2008). Lifestyle- and diet-related factors in late-life depression-a 5-year follow-up of elderly European men: the FINE study. International fournal of Geriatric Psychiatry, 23, 478-484. doi: 10.1002/gps.1919

Cao, P. Y., Luo, H. Q., Hou, L. S., Yang, X. X. and Ren, X. H. (2016). [Depressive Symptoms in the Mid- and Old-aged People in China]. Sichuan Da Xue Xue Bao Yi Xue Ban, 47, 763-767.

Cassidy, K. et al. (2004). Association between lifestyle factors and mental health measures among communitydwelling older women. Australian and New Zealand fournal of Psychiatry, 38, 940-947. doi: 10.1080/j.1440-1614.2004 .01485.x

CHARLS. (2018). About CHARLS. Available at: http://charls .pku.edu.cn/en/page/about/CHARLS; last accessed 9 September 2019.

CHARLS Research Team. (2013). Challenges of population aging in China: Evidence from the national baseline survey of the China Health and Retirement Longitudinal Study. Available at: http://bw.dowjones.com/ public/resources/documents/charls0530.pdf; last accessed 9 September 2019.

Chin, W. Y., Choi, E. P. and Wan, E. Y. (2016). Trajectory pathways for depressive symptoms and their associated factors in a Chinese Primary Care Cohort by growth mixture modelling. PLoS One, 11, e0147775. doi: 10.1371/journal.pone.0147775

Crowley, S. L. and Fan, X. (1997). Structural equation modeling: Basic concepts and applications in personality assessment research. Fournal of Personality Assessment, 68, 508-531. doi: 10.1207/s15327752jpa6803_4

Dai, H. L. et al. (2019). Association between social health status and depressive symptoms among communitydwelling elderly adults in Zhejiang Province, China. fournal of Zhejiang University Science B, 20, 910-919. doi: 10.1631/jzus.B1900207
De La Torre-Luque, A. et al. (2019). Long-term trajectories of depressive symptoms in old age: relationships with sociodemographic and health-related factors. Fournal of Affective Disorders, 246, 329-337. doi: 10.1016/j.jad.2018.12.122

Doraiswamy, P. M., Khan, Z. M., Donahue, R. M. and Richard, N. E. (2002). The spectrum of quality-of-life impairments in recurrent geriatric depression. Fournals of Gerontology Series A-Biological Sciences and Medical Sciences, 57, M134-M137. doi: 10.1093/gerona/57.2.m134

Fatima, M., Sehar, A., Ali, M., Iqbal, A. and Shaukat, F. (2019). Incidence of Depression Among Community Dwelling Healthy Elderly and the Predisposing Socioenvironmental Factors. Cureus, 11, e4292. doi: 10.7759/ cureus. 4292

Gao, M. et al. (2017). Does an empty nest affect elders' health? Empirical evidence from China. International fournal of Environmental Research and Public Health, 14, e463. doi: $10.3390 /$ ijerph 14050463

Gao, S. et al. (2009). Correlates of depressive symptoms in rural elderly Chinese. International fournal of Geriatric Psychiatry, 24, 1358-1366. doi: 10.1002/gps.2271

Gazalle, F. K., Lima, M. S., Tavares, B. F. and Hallal, P. C. (2004). [Depressive symptoms and associated factors in an elderly population in southern Brazil]. Revista Saude Publica, 38, 365-371. doi: 10.1590/s003489102004000300005

Gibson, R. C., Neita, S. M., Abel, W. D., James, K. and Eldemire-Shearer, D. (2013). Sociodemographic factors associated with depressive symptoms among elderly persons from two communities in Kingston, Jamaica. West Indian Medical fournal, 62, 615-619. doi: 10.7727/wimj .2012 .273

Glaesmer, H., Riedel-Heller, S., Braehler, E., Spangenberg, L. and Luppa, M. (2011). Age- and gender-specific prevalence and risk factors for depressive symptoms in the elderly: a population-based study. International Psychogeriatrics, 23, 1294-1300. doi: 10.1017/ S1041610211000780

Graham, K., Massak, A., Demers, A. and Rehm, J. (2007). Does the association between alcohol consumption and depression depend on how they are measured? Alcoholism-clinical and Experimental Research, 31, 78-88. doi: 10.1111/j.1530-0277.2006.00274.x

Guimaraes, L. A. et al. (2019). Depressive symptoms and associated factors in elderly long-term care residents. Cien Saude Colet, 24, 3275-3282. doi: 10.1590/141381232018249.30942017

Hackett, M. L., Yapa, C., Parag, V. and Anderson, C. S. (2005). Frequency of depression after stroke: a systematic review of observational studies. Stroke, 36, 1330-1340. doi: 10.1161/01.STR.0000165928.19135.35

Haigh, E. A. P., Bogucki, O. E., Sigmon, S. T. and Blazer, D. G. (2018). Depression among older adults: a 20-year update on five common myths and misconceptions. American fournal of Geriatric Psychiatry, 26, 107-122. doi: 10.1016/j.jagp.2017.06.011

Hajat, S., Haines, A., Bulpitt, C. and Fletcher, A. (2004). Patterns and determinants of alcohol consumption in people aged 75 years and older: results from the MRC trial of assessment and management of older people in the community. Age and Ageing, 33, 170-177. doi: 10.1093/ ageing/afh046 
Hsiao, Y. C. and Chen, C. Y. (2018). Exploring Individual, family, and extrafamilial factors associated with depression among elderly residents of care settings. International Fournal of Aging and Human Development, 86, 219-241. doi: 10.1177/0091415017699938

Hu, L.-T. and Bentler, P. M. (1999). Cutoff criteria for fit indexes in covariance structure analysis: conventional criteria versus new alternatives. Structural Equation Modeling, 6, 1-55. doi: 10.1080/10705519909540118

Huang, Q. B., Wang, X. H. and Chen, G. (2015). Reliability and validity of 10-item CES-D among middle aged and older adults in China. China fournal of Health Psychology, 23, 1036-1041. doi: 10.13342/j.cnki.cjhp.2015 .07 .023

Hyland, A. et al. (2004). Predictors of cessation in a cohort of current and former smokers followed over 13 years. Nicotine and Tobacco Research, 6, S363-S369. doi: 10.1080/ 14622200412331320761

Ichimori, A., Tsukasaki, K. and Koyama, E. (2015). Illuminance, subjective sleep quality, and psychosomatic health in elderly individuals requiring care: a survey of Japan's Hokuriku Region in Winter. Fournal of Community Health Nursing, 32, 104-114. doi: 10.1080/07370016 .2015 .1026158

Islam, A. and Smyth, R. (2015). Do fertility control policies affect health in old age? Evidence from China's one-child experiment. Health Economics, 24, 601-616. doi: 10.1002/ hec. 3047

Javed, S. (2014). Role of sociocultural factors in depression among elderly of Twin Cities (Rawalpindi and Islamabad) of Pakistan. Current Gerontology and Geriatrics Research, 2014, 230737. doi: $10.1155 / 2014 / 230737$

Kaneko, Y., Motohashi, Y., Sasaki, H. and Yamaji, M. (2007). Prevalence of depressive symptoms and related risk factors for depressive symptoms among elderly persons living in a rural Japanese community: a cross-sectional study. Community Mental Health fournal, 43, 583-590. doi: 10.1007/s10597-007-9096-5

Katz, S., Ford, A. B., Moskowitz, R. W., Jackson, B. A. and Jaffe, M. W. (1963). Studies of illness in the aged. The index of adl: a standardized measure of biological and psychosocial function. $\mathcal{F} A M A, 185,914-919$. doi: 10.1001 jama.1963.03060120024016

Kendler, K. S. et al. (1993). Smoking and major depression. A causal analysis. Archives of General Psychiatry, 50, 36-43. doi: 10.1001/archpsyc.1993.01820130038007

Kim, H. K. and Mckenry, P. C. (2002). The relationship between marriage and psychological well-being: a longitudinal analysis. Fournal of Family Issues, 23, 885-911. doi: $10.1177 / 019251302237296$

Luijendijk, H. J., Stricker, B. H., Hofman, A., Witteman, J. C. and Tiemeier, H. (2008). Cerebrovascular risk factors and incident depression in community-dwelling elderly. Acta Psychiatrica Scandinavica, 118, 139-148. doi: 10.1111/j.1600-0447.2008.01189.x

Manber, R. and Chambers, A. S. (2009). Insomnia and depression: a multifaceted interplay. Current Psychiatry Reports, 11, 437-442.

National Bureau of Statistics. (2019). Age composition and dependence ratio of population, national data. Available at:
http://data.stats.gov.cn/english/easyquery.htm?cn=C01; last accessed 9 Semptember 2019.

Ni, Y., Tein, J. Y., Zhang, M., Yang, Y. and Wu, G. (2017). Changes in depression among older adults in China: a latent transition analysis. Fournal of Affective Disorders, 209, 3-9. doi: 10.1016/j.jad.2016.11.004

Pentz, M. A. and Chou, C. P. (1994). Measurement invariance in longitudinal clinical research assuming change from development and intervention. Fournal of Consulting and Clinical Psychology, 62, 450-462. doi: 10.1037//0022006x.62.3.450

Rodriguez, C. A., Schonfeld, L., King-Kallimanis, B. and Gum, A. M. (2010). Depressive symptoms and alcohol abuse/misuse in older adults: results from the Florida BRITE Project. Best Practice and Mental Health, 6, 90-102.

Solmi, M. et al. (2019). Network analysis of the relationship between depressive symptoms, demographics, nutrition, quality of life and medical condition factors in the Osteoarthritis Initiative database cohort of elderly NorthAmerican adults with or at risk for osteoarthritis. Epidemiology and Psychiatric Sciences, 29, e14. doi: 10.1017/ S204579601800077X

Staner, L. (2010). Comorbidity of insomnia and depression. Sleep Medicine Reviews, 14, 35-46. doi: 10.1016/j.smrv.2009 .09 .003

Su, H., Cao, J., Zhou, Y., Wang, L. and Xing, L. (2018). The mediating effect of coping style on personality and mental health among elderly Chinese empty-nester: a cross-sectional study. Archives of Gerontology and Geriatrics, 75, 197-201. doi: 10.1016/j.archger.2018.01.004

Taheri Tanjanai, P., Moradinazar, M. and Najafi, F. (2017). Prevalence of depression and related social and physical factors amongst the Iranian elderly population in 2012. Geriatrics and Gerontology International, 17, 126-131. doi: 10.1111 ggi.12680

Tanaka, H., Sasazawa, Y., Suzuki, S., Nakazawa, M. and Koyama, H. (2011). Health status and lifestyle factors as predictors of depression in middle-aged and elderly Japanese adults: a seven-year follow-up of the Komo-Ise cohort study. BMC Psychiatry, 11, 20. doi: 10.1186/1471244X-11-20

Tsai, A. C., Chi, S. H. and Wang, J. Y. (2013). Crosssectional and longitudinal associations of lifestyle factors with depressive symptoms in $>/=53$-year old Taiwanese results of an 8-year cohort study. Preventive Medicine, 57, 92-97. doi: 10.1016/j.ypmed.2013.04.021

Urbina Torija, J. R., Flores Mayor, J. M., Garcia Salazar, M. P., Torres Buisan, L. and Torrubias Fernandez, R. M. (2007). [Depressive symptoms in the elderly. Prevalence and associated factors]. Gaceta Sanitaria, 21, 37-42. doi: 10.1157/13099119

Van Orden, K. A., Chen, S., O'Riley, A. and Conwell, Y. (2015). Course of late-life depression in China is chronic and unremitting. International fournal of Geriatric Psychiatry, 30, 409-415. doi: 10.1002/gps.4151

Weinger, K. and Lee, J. (2006). Psychosocial and psychiatric challenges of diabetes mellitus. Nursing Clinics of North America, 41, 667-680. doi: 10.1016/j.cnur.2006.07.002

Wen, Y., Liu, C., Liao, J., Yin, Y. and Wu, D. (2019). Incidence and risk factors of depressive symptoms in 4 years 
of follow-up among mid-aged and elderly communitydwelling Chinese adults: findings from the China Health and Retirement Longitudinal Study. BMF Open, 9, e029529. doi: 10.1136/bmjopen-2019-029529

Xie, L. Q., Zhang, J. P., Peng, F. and Jiao, N. N. (2010). Prevalence and related influencing factors of depressive symptoms for empty-nest elderly living in the rural area of YongZhou, China. Archieves of Gerontology and Geriatrics, 50, 24-29. doi: 10.1016/j.archger.2009 .01 .003

Yang, J. et al. (2018). The Association between social contacts and depressive symptoms among elderly Koreans. Psychiatry Investigation, 15, 861-868. doi: 10.30773/pi .2018.06.28.1

Yang, Z., Chen, R., Hu, X. and Ren, X. H. (2017). [Factors that related to the depressive symptoms among elderly in urban and rural areas of China]. Zhonghua Liu
Xing Bing Xue Za Zhi, 38, 1088-1093. doi: 10.3760/cma.j .issn.0254-6450.2017.08.018

Yun, M. and Kim, E. (2020). Gender difference in the association between alcohol consumption and depressive symptoms among the elderly in rural areas. Fournal of Ethnicity in Substance Abuse. doi: 10.1080/15332640.2019 .1704336

Zalavadiya, D. D. et al. (2017). A comparative study of depression and associated risk factors among elderly inmates of old age homes and community of Rajkot: a Gujarati Version of the Geriatric Depression Scale-Short Form (GDS-G). Indian Fournal of Community Medicine, 42, 204-208. doi: 10.4103/ijcm.IJCM_181_16

Zhang, C. et al. (2019). Prevalence and related influencing factors of depressive symptoms among empty-nest elderly in Shanxi, China. Fournal of Affective Disorders, 245, 750-756. doi: $10.1016 /$ j.jad.2018.11.045 